\title{
IEC 61850 SCL Validation Using UML Model in Modern Digital Substation
}

\author{
Byungtae Jang, Alidu Abubakari, Namdae Kim \\ Power System Technology Group, Korea Electric Power Corporation, Daejeon, South Korea \\ Email: byungtae.jang@kepco.co.kr, a abubakari32@yahoo.com, namdae.kim@kepco.co.kr
}

How to cite this paper: Jang, B.-T., Abubakari, A. and Kim, N. (2018) IEC 61850 SCL Validation Using UML Model in Modern Digital Substation. Smart Grid and Renewable Energy, 9, 127-149. https://doi.org/10.4236/sgre.2018.98009

Received: August 7, 2018

Accepted: August 27, 2018

Published: August 30, 2018

Copyright $\odot 2018$ by authors and Scientific Research Publishing Inc. This work is licensed under the Creative Commons Attribution International License (CC BY 4.0).

http://creativecommons.org/licenses/by/4.0/ (c) (i) Open Access

\begin{abstract}
The IEC 61850 standard stipulates the Substation Configuration Description Language (SCL) file as a means to define the substation equipment, IED function and also the communication mechanism for the substation area network. The SCL is an eXtensible Markup Language (XML) based file which helps to describe the configuration of the substation Intelligent Electronic Devices (IED) including their associated functions. The SCL file is also configured to contain all IED capabilities including data model which is structured into objects for easy descriptive modeling. The effective functioning of this SCL file relies on appropriate validation techniques which check the data model for errors due to non-conformity to the IEC 61850 standard. In this research, we extend the conventional SCL validation algorithm to develop a more advanced validator which can validate the standard data model using the Unified Modeling Language (UML). By using the Rule-based SCL validation tool, we implement validation test cases for a more comprehensive understanding of the various validation functionalities. It can be observed from the algorithm and the various implemented test cases that the proposed validation tool can improve SCL information validation and also help automation engineers to comprehend the IEC 61850 substation system architecture.
\end{abstract}

\section{Keywords}

IEC 61850, Substation Automation, IED, XML, UML, XMI, Schema, Rule-Based SCL Validation, Syntax, Semantic Data Model, SCL Editor

\section{Introduction}

Power systems are complex systems which electrically connect geographically dispersed equipment from the power plants to the transmission and distribution substations and finally end with the supply of electricity to the customer [1]. 
Various devices and operating systems from power plants to consumers have been introduced and utilized depending on the characteristics of such equipment. "Stand Alone" devices based on traditional standards are highly dependent on the technology of the manufacturer. Therefore, it is very difficult to integrate various devices into the complex system in order to implement them, and to be able to do that various types of interface technologies are required. Since the advent of Smart Grid technology, we have created a common standard through international standardization rather than the manufacturer-dependent technology, leading to the establishment of a system-oriented technology environment. In particular, the IEC 61850 international standard proposes communication network and data standardization for the automation of power system [2] [3] [4] [5]. In IEC 61850 Edition 1.0, standardization was limited to the substations domain while IEC 61850 Edition 2.0 extends the automation to all areas of power system [6].

In order to standardize the substation data model, the devices operating in the power system are identified and their related data is classified into the property class [7]. For example, in the substation domain, a circuit breaker is used to quickly disconnect a fault section in the event of a fault in a system. The breaker is classified as Logical Node by XCBR and the data model name is specified. The detailed attributes are classified into Data Objects and Data Attributes, and data names are assigned. In this way, data model standardization work was carried out and a large amount of data was processed in a text-based table format. However, the use of text-based tables has not been able to prevent duplication causing inconsistencies in the standardization of large amounts of data. Especially as the application domain of power system is expanded. When the IEC 61850 Edition 2.0 document was developed, the number of Logical Nodes defined in IEC 61850 Edition 1.0 has increased rapidly from 90 to 300 (over 200\%). To address this challenge, the IEC 61850 International Standardization Work Group, standardized the data models using UML (Unified Modeling Language) to improve upon the manual text-based table formatting of the standard data model [8].

The UML model supports XML Metadata Interchange (XMI) for the interchange of UML metadata between systems. In this paper, we propose a technique for verifying standard data compliance for arbitrary IEC 61850 based devices using UML and XMI. Current SCL validators require manual update of the data model from an external database in order to maintain up-to-date validation functionality. Our proposed tool uses the UML model which can update it data model automatically in order to implement data consistency checks on the SCL file. Also it used the rule-based validation which would help the test engineer understand the cause of an error and how to resolve it. This paper is structured as follows; Section I reviews basic IEC 61850 concepts including the importance of the standard while Section II discusses the overview of both the SCL engineering in digital substation and also the IEC 61850 data modelling approach 
using the metamodeling technique. Section III then elaborates on the concept of data model validation in IEC 61850. This includes a discussion on the conventional SCL validation algorithm and a detail presentation on the proposed UML based SCL data model validator. We present on the practical implementation procedure, main features of the tool, test scenario, actual implementation, error checking mechanism in section IV. The advantages of the proposed validation are also discussed in Section IV and in Section V we present the conclusion.

\section{Literature Review}

Various researches have been conducted on substation configuration and SCL since the conception of Substation Automation (SA) System. Most existing research focuses on the design of vendor independent configuration tool for SCL including [9] and [10]. Research in [9] studied the use of the SCL in describing IEC 61850 models. The paper investigated the application of SCL in the description of substation domain objects and models. The authors also studied on the differences and similarities between the various SCL files and also constraints in practical application of the SCL in a digital substation. Research in [10] implemented a visual designer to facilitate the engineering of SCL files without the need for knowing the details of the underlying XML syntax. The researchers developed a graphic model approach using the UML model to make it simple for engineers to configure the SCL file. Research work by [11] also focused on the development of fully graphical XML-based SCL configurator by taking SCL object model, visual representation of substation elements into consideration. Their work proved the possibility of integrating the XML-based Scalable Vector Graphics (SVG) into SCL visual representation. The work in [12] focused on the use of SQL server to design and implement an SCL configurator. The basic idea is the transformation of data into XML format using a relative method.

A comparative approach for the differentiation of smart substation SCL configuration files is presented in [13]. In addition, [14] researched the possibility of mapping SCL to ArchiMate for easy comprehension and to support easy integration with an IEC 61850 based SA System. ArchiMate is an open meta-modeling standard which supports stakeholders in understanding the integration of ICT systems to power transmission. An extension of the IEC 61850 SCL Configuration file to gateway is provided in [15]. The work in [16] presented a simplified beyond schema validation of the SCL file by introducing the machine based validation of notations, requirements and/or conventions. This work gives a good comprehensive approach towards understanding the need for validation and the basics of validation. With respect to SCL validators, there is the command line validation for XML based schema and XML based editors which supports schema validation. Some vendor specific tools exist too but are quite expensive and do not support some functionalities [17]. These functionalities include the lack of support for the UML model which ensures data consistency and also the use of the rule validation which makes error correction easier due to 
hyperlink between error type and line of SCL XML file where it occurred. These conventional SCL tools operate using the "black-box" concept whereby the user does not usually understand what the error means and how to correct it. Some tools are also integrated into manufacture-specific SCL configuration tools making it difficult to use without the right expertise. There is the need to implement manufacturer independent validator which verifies the SCL file beyond schema. Our previous work in [18], gives an outlook on the concept of SCL validation and also the aspects to be validated. We presented a simple and comprehensive engineering tool design which would facilitate the development of an intelligent substation system via a thorough SCL verification process. This work is an extension of the work in [18] where we explore the architecture and implementation of the SCL data verification component of the tool.

\section{IEC 61850 Metadata Architecture}

Metadata refers to the used to describe another related data. The IEC 61850 standard utilizes the metamodeling approach to conceptualize the domain data type model which defines the semantics of all the data communicates in the substation. Thus the semantics of the data is modelled by metadata which are parts of the metamodel [19]. The IEC 61850 metamodeling which refers to the abstract description of data using data can be seen in Figure 1. The metamodel abstracts the concept of Logical node and the data attributes which are not application-domain specific. The Domain Type Model abstracts data model defined for substation specific logical nodes and data attributes. The data instance model represents the instance of the domain data type used in the IED. The advantage of using the data metamodel is that it helps manage the large amount of power substation data and data integration [19].

\subsection{Hierarchical IEC 61850 Metadata Modeling Technique}

The data model represents different aspects of the substation function including its associated data model and communication services which helps in the comprehension of the real-world inter-device interaction. The data model that is typically exchanged between multi-vendor devices is well defined in part 7 of the IEC 61850 standard [20]. The SA system produces large volumes of data in real-time necessary for continuous monitoring of its performance. The basic level of information consists of data from devices connected to the process including primary equipment. This produces raw process-level data made up of status information, measurement values and control information which is communicated across distributed IEDs by way of busses for effective system health monitoring. The primary data collected from process-level devices are processed into secondary data and reported to the SCADA for further analysis and response. The basic abstraction of the data model which exchanges data is the Logical Node (LN). An example is the XCBR LN for circuit breaker operation in an IEC 61850 compliant IED as shown in Figure 2. 


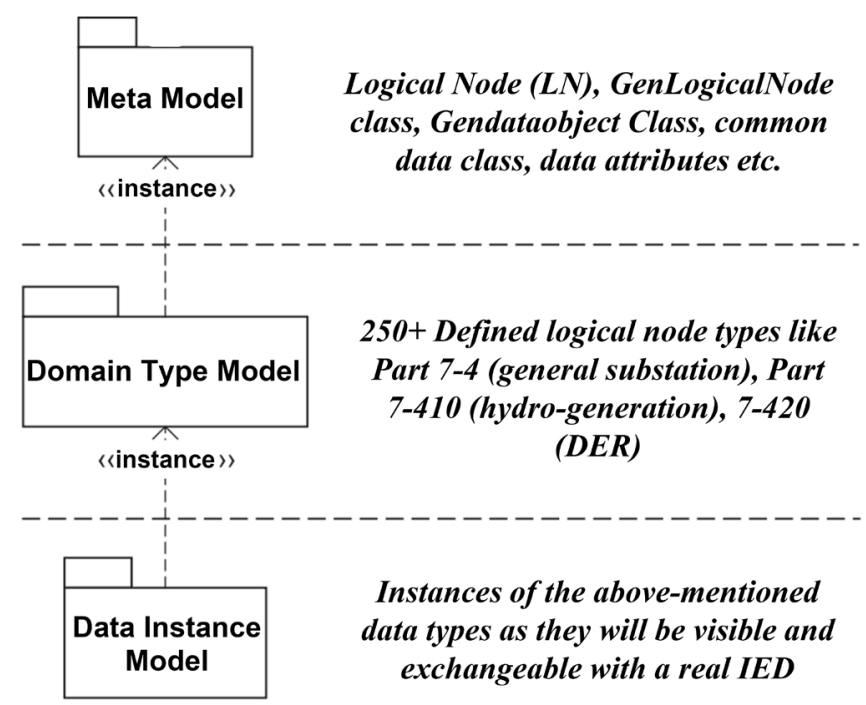

Figure 1. IEC 61850 Meta modelling architecture.

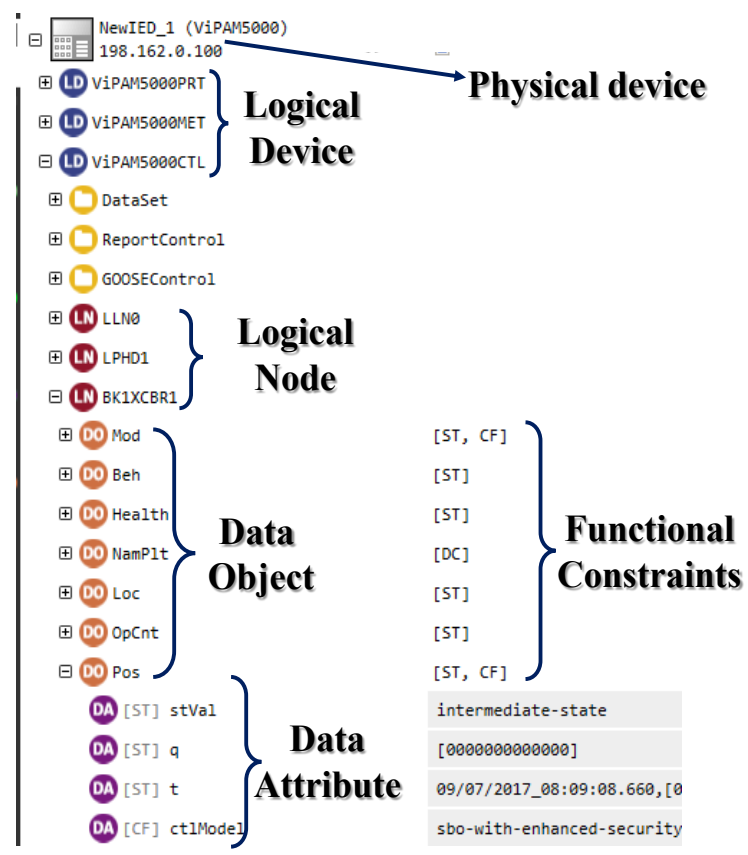

Figure 2. Hierarchical data modeling case for IEC 61850.

A group of LN ( $L L N O, L P H D$, and XCBR) is used to build a logical device (LD) while a group of LD (ViPAM5000PRT, ViPAM5000MET and Vi$P A M 5000 C T L)$ is implemented as the physical device or the IED. The XCBR LN decomposes into various data objects including Mode (Mod), Beh (Behavior), NamePlt (Nameplate), Loc (local Control behavior), OpCnt (Operation Counter) and Pos (switch Position). The switch position "Pos" data object is also made up of groups of data attributes including control data attributes, substitution data attributes and others like configuration, description and extension data attributes. The "Pos" data object in Figure 2, has about four (4) data attributes for XCBR LN. The data attribute "Pos.ctlval" is used for controllable informa- 
tion like setting circuit breaker "on" or "off" while "Pos.stVaP" is used to communicate the condition (status). According to the standard, the condition of the circuit breaker can be "on", "off", "bad-state" and "intermediate-state". The "Pos.q" is used as an indication of the validity of the status value while "Pos. $t$ " represents the time of the last change of the status value. The position switch (Pos) is a Double Point Controllable (DPC) Common Data Class (CDC).

\subsection{Representation of IEC 61850 Metadata in the Standard}

The hierarchical meta model architecture of the IEC 61850 data model is represented using tables in both edition 1 and 2 of the standard. An example of such metamodeling of substation circuit breaker data is shown in Figure 3.

As can be observed from Figure 3, the Logical Node (LN) Class, Data Object (DO), Data Attributes (DA), Functional Constraints (FC), data type and the presence conditions are defined by the IEC 61850 in tables [21] [22]. The IEC 61850 edition 1 uses text-based description of the data and the meta of the data model semantics and these are structured by means of table. Sometimes, these tables also reference other tables in other documents without explicit navigation links between them. This makes the tabular-approach to data model structuring very complicated with over 150 tables scattered within 600 pages of 5 different IEC 61850 standards. To address this problem created by too many text-based data model description, the UML model has been proposed [19]. The UML model allows for consistent data modelling across multiple application using the metamodeling approach while establishing explicit relationships though inheritance properties [23] [24]. It also promotes processability through the use of computer-aided solutions to automatically generate codes, check consistency, and also generate text documents. An example of the UML model for the circuit breaker is shown in Figure 4.

Modeling in UML allows the use of cardinality and optionality instead of multiple graphical symbols. This eases the complications in the design process and also the model comprehension. Ambiguities in the standard definition can be identified easily due to the reduction in the number of tables used for data type descriptions. Also, ambiguity in standard interpretation by device manufacturers and researchers can be mitigated. The use of the UML model for IEC 61850 data description also ensures comprehension of the standard data model thereby promoting effective communication between software architects and domain engineers.

\section{Overview of SCL in IEC 61850 Digital Substation}

In order to achieve the complete interoperability function among different IED manufacturers, there is the need to define a standardized method for data exchange and also standard exchangeable data objects. A common method to achieve this is by adopting the SCL [25]. The SCL uses the XML as a meta-language to describe the IED, the substation structure and its communication 


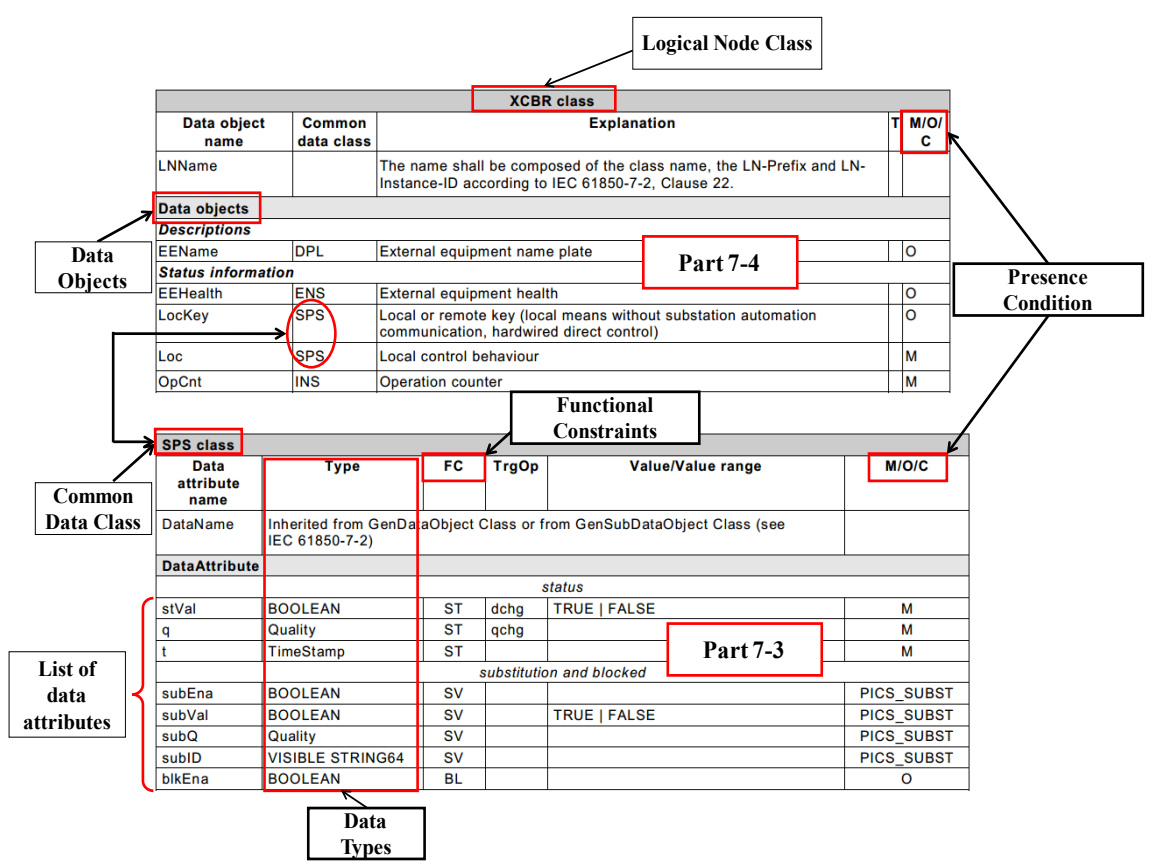

Figure 3. Hierarchical data modeling case for IEC 61850.

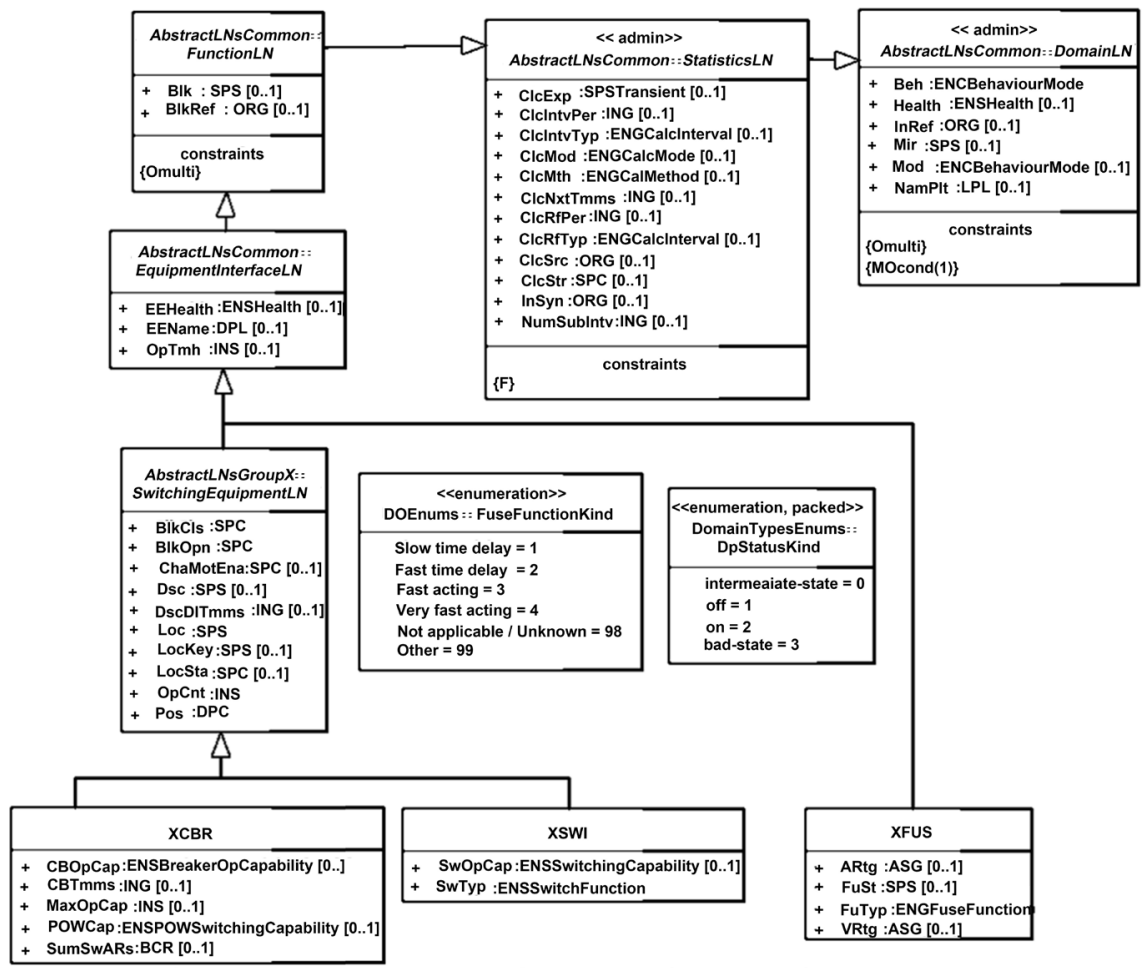

Figure 4. IEC 61850 UML diagram for circuit breaker (XCBR).

network. Description of the IED includes its configuration logic nodes, data and the data attributes. SCL simplifies and automates the configuration of the digital substation system, thus increasing the flexibility of information exchange between SA systems and/or devices. 
This reduces the workload, the rate of error occurrence and thus increase overall power efficiency. The complete description of the SA System as can be seen in Figure 5 occurs under five (5) elements in the SCL file. These are the header, substation, IED, Communication system and DataType Templates. The header element includes the identification of the SCL file, its version and revision information and options for mapping names to signals. The substation element describes the functional architecture of the substation including the primary devices and the associated electrical connects. The IED element describes the IED configuration including the logical devices and its associated logical nodes and access points. It defines the IED communication services, its logical node type, instantiated data and the configuration values. The communication element defines the means of connections between logical nodes via subnetworks and access points. Finally, the datatype template defines the instantiable logical node type, data object type, data attribute type and enumerate data type for each logical device associated [26].

\subsection{General Procedure for SCL Engineering}

To make the content easier to understand and use, the IEC 61850 standard defines six (6) files each containing different information for different applications in the engineering process. These files are the IED Capability Description (ICD), System Specification Description (SSD), System Configuration Description (SCD), the Configured IED Description (CID), the Instantiated IED Description (IID) and the System Exchange Description (SED). The ICD file contains the information concerning the IED capabilities, the system description, and the default factory-set communication description of the IED. The SSD describes the substation architecture using the single line diagram and thus helps dictate the

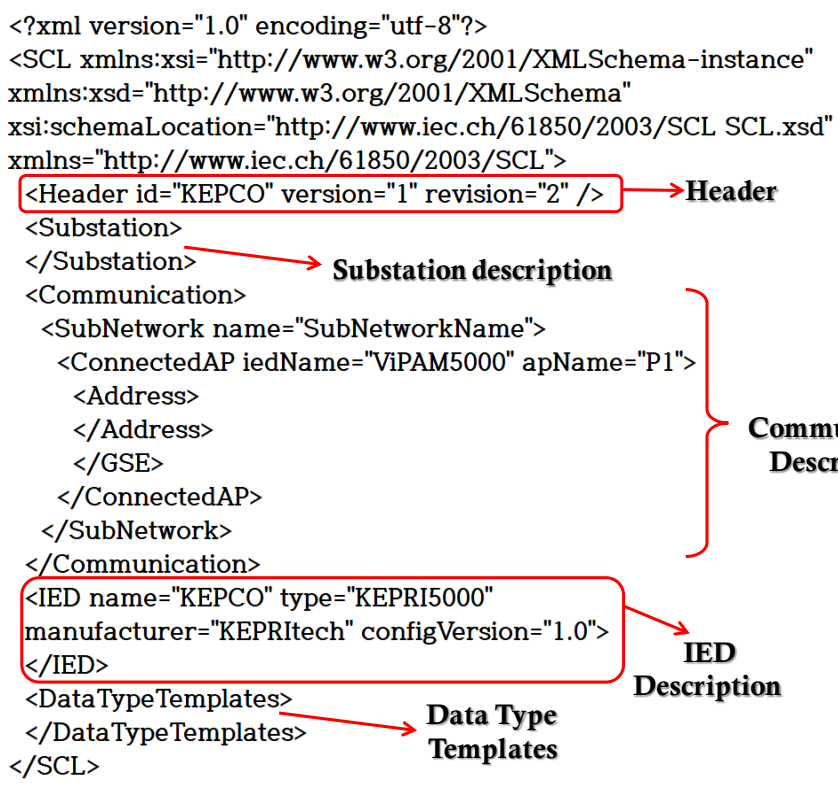

Figure 5. The various sections of a typical SCL file. 
required logical nodes for the SA system. The SCD file contains specifications of the substation architecture with their allocated logical nodes and communication services and interface. The CID file is used to configure a particular vendor IED in the SA project. The second edition defines the IID file which contains the information of an already pre-configured instance of an IED including project addresses and datasets. This can be used to configure a new IED's ICD file especially in the case of substation expansion. To identify the interface between different projects, the SED file can be used. The substation specification design is loaded from its database into the system specification configurator to produce a complete single-line description of the substation in the form of the .SSD file. The ICD files are then generated by loading the IED capabilities from its associated Database into the manufacturer-specific IED Configurator. The SSD files and the ICD files are imported into the vendor-independent system configuration tool which generates the SCD file. After the generation of the SCD file, all IEDs would be associated with specific field equipment and independent processes function. This implies all logical devices, logical nodes and data objects are bound to real-time processes and functions. Lastly, the CID files are created and imported to each IED from the vendor-specific IED configurator [25]. The complete engineering process for the various SCL files is shown in Figure 6.

\subsection{Importance of SCL in Digital Substation}

The SCL file is a critical element in the complete life-cycle of an SA system including the design, commissioning, testing and evaluation etc. In order to implement a simple and easy technique for the exchange of data between engineering tools at various levels of the SA system, it is important to design a single file format for all devices. This is achieved using the SCL file as it can in its design process take into account the different and sometimes proprietary data definitions and data format. The SCL file contains the static description of an IED which is IEC 61850 compatible. It helps to describe the capabilities of the IED device. It can also serve as a means to document the complete system for easy

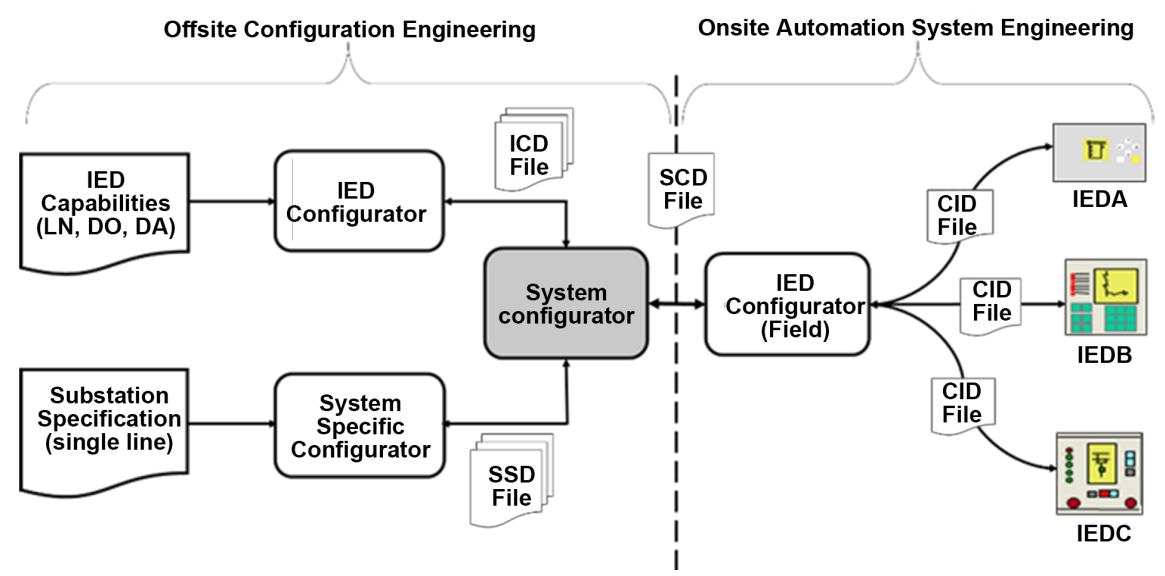

Figure 6. IEC 61850 SCL engineering process. 
analysis especially during extensions and retrofitting. By developing a database library of substation configuration in the form of single line diagram, it is possible to easily adjust the substation system design to check for compatibility and performance measurement before actual implementation. The SCL file can be used to generate and analyze the semantic model of the IED under consideration with or without reference to manufacturers' documentation.

The SCL file can also be used to retrieve a section of the IED using the self-description capability of the IED for verification against the standard. It can serve as a means to create a virtual IED on a computer using the appropriate simulation tools. It can be used in conjunction with the appropriate simulation tools to test various communication scenarios and techniques which cannot be implemented physically due to the cost involved.

\subsection{The Concept of SCL Validation}

SCL validation can be defined as the tools and techniques required to check the conformity of an IED SCL file to the IEC 61850 standard to enable interoperable operation with other devices in the modern digital substation. It is a testing tool designed to enable the verification of the SCL file both syntactically and semantically in accordance with IEC 61850 standard part 6. In order to perform this verification, the validation tool is required to extract the schema (syntax) and the data model of the SCL file. The ideal SCL validator is also required to easily import all related SCL files including SCD, SSD, SED, CID, IID and ICD while providing a database of the standard data model for comparison. Multivendor IEDs are expected to communicate and work successfully in a plug and play fashion if their SCL files are successfully validated [27]. Therefore, there is the need to design an effective SCL validation tool which is the main focus of this research paper. The operational method of the SCL validator is illustrated in Figure 7 based on discussions in [16]. The comparative analyzer in simple terms compares the object model of the SCL file to the standard rules in IEC 61850 . Any inconsistency results in a failed output which would alert the engineer of a possible interoperability problem before the actual implementation and commissioning of the IEDs. This would prevent the future malfunctioning of the SA system.

\section{Design Concept for IEC 61850 Data Model Validation}

\subsection{Conventional SCL Tool Design}

The existing architecture for SCL validation tool is shown in Figure 8. The data model database is extracted manually from the IEC 61850 standard (typically from part 7). This method of developing the data model DB is prone to errors due to human involvement.

Also the testing is time consuming and data model database would require constant changes or updates due to the frequent addition of different domain data types. Also it is difficult to main the relationship between the various data 


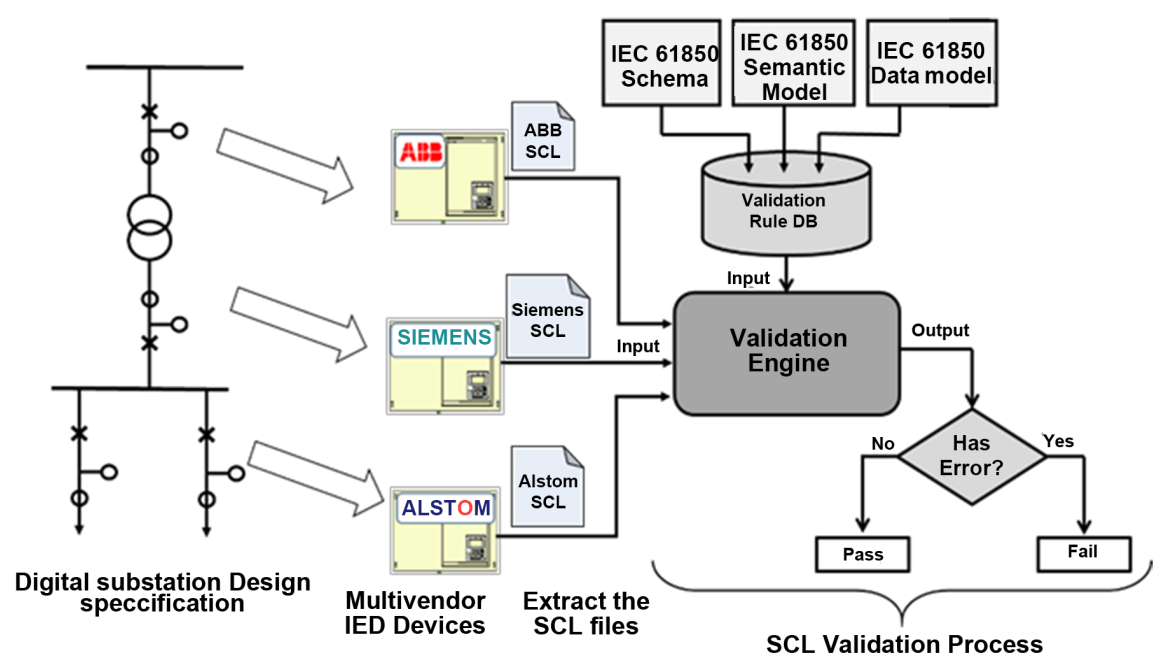

Figure 7. Illustration of the method of execution of an SCL validator.

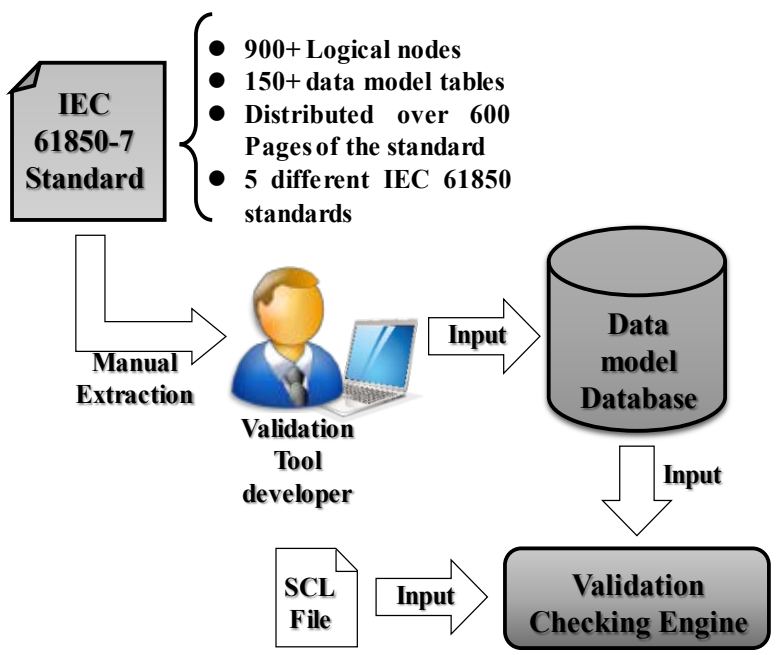

Figure 8. Manual extraction of the data model from the IEC 61850 database.

types and data models due to the "flatness" of the database. Basically this approach is inefficient and there is a need to develop a new approach to validate the data model of the SCL file under test.

\subsection{Proposed SCL Validation Tool Design}

The algorithm of the proposed SCL validation tool which uses the UML database is as shown in Figure 9. The UML model is imported into the validation system where it is converted to XMI and to the relational database for easy access. The guide DB is a database of all the pre-defined validation rules. The test engineer can select multiple rules for the validation. The validation engine is responsible for extracting the SCL data model, and ensuring a thorough check of the data using the validation rule based on the data extracted from the UML model by way of XMI database. In case an error is discovered during the validation, an 


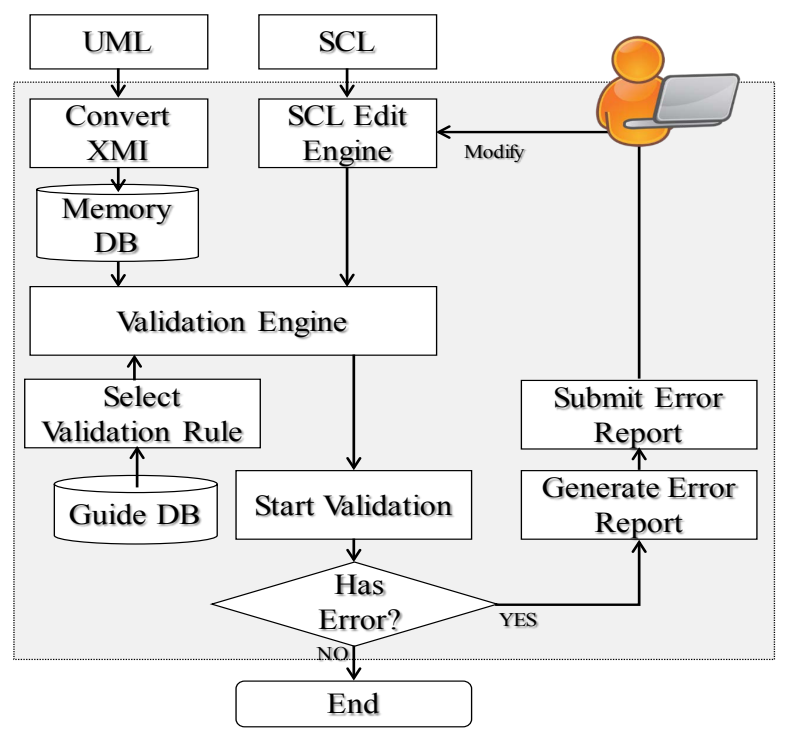

Figure 9. Proposed Algorithm for the IEC 61850 data model validation.

error report is submitted to the test engineer for further evaluation and correction using the SCL edit engine.

\section{UML Transformation to Database}

The SCL validation system imports the XMI (XML Metadata Interchange) from the UML file (.eap) in order to generate the IEC 61850 data model database required for the SCL validation. The process is illustrated in Figure 10.

This transformation from UML to XMI is enabled automatically by the software-based model UML driven compiler like the Sparx Enterprise architecture software. The UML model describes objects using classes, attributes, associations, actors, messages, packages, collaborations, states and other concepts standardized in the UML language while the XMI standard defines the algorithm for use in the generation of metamodel vocabulary using XML tags. This implies that the XMI uses XML classes, attributes and associations to define a complete $\mathrm{XML}$ vocabulary for UML. The XMI analysis tool is responsible for ensuring the syntactic completeness of the exported XMI file utilizing a fixed set of declaration used by all XMI documents. It also uses the standard IEC 61850 hierarchical data structure to extract the XMI database by grouping all data under specific categories.

An example of the XMI database categorization is shown in Figure 11. This is then exported to the SCL validator memory database where the data is stored using relational database architecture. Since the memory database is relational, it contains tables and columns for each XML-tag data which in this case represents the XMI database categorization shown in Figure 11. The use of the UML model for IEC 61850 data modelling helps achieve data consistency through maintaining relationship between the various data types. This relationship, which is key to establishing data consistency, is also maintained through the use of the 


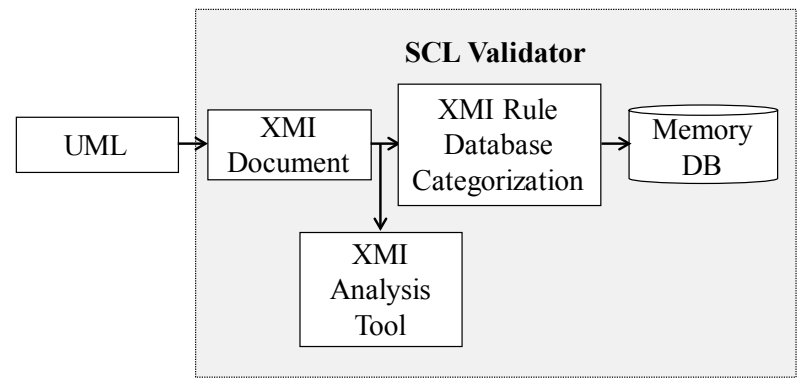

Figure 10. Proposed Algorithm for the IEC 61850 data model validation.

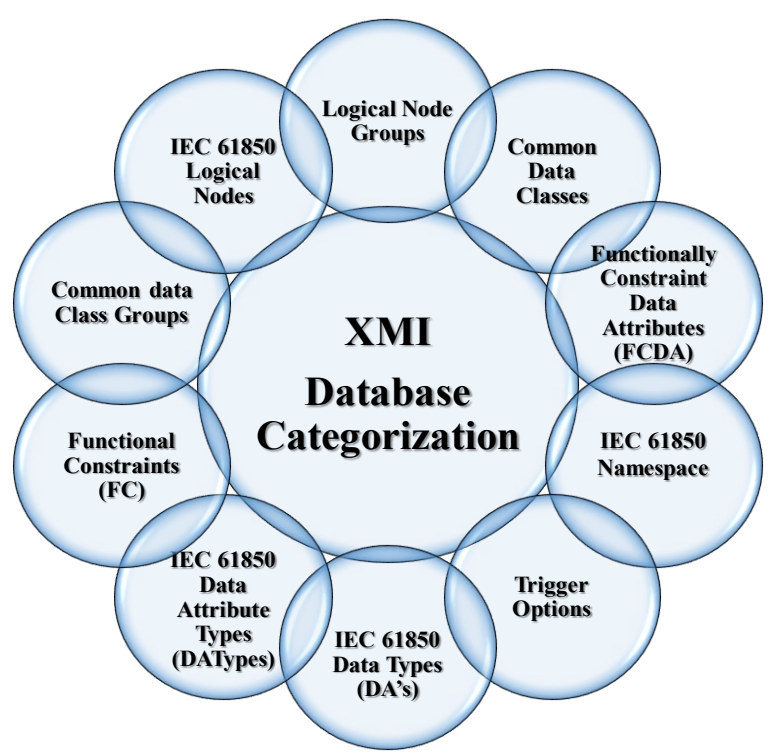

Figure 11. Categories for grouping the UML data model information extracted from XMI.

relational database for the SCL validator memory database. This is because the relational database architecture separates the data into its different constituents while still maintaining relationship between them.

An example is seen in Figure 12 the XMI analysis tool extracts the XMI database by grouping all IEC 61850 Logical Node Groups, all standard IEC 61850 Logical Nodes, Common data classes, Common data class groups, Functionally Constraint Data Attributes (FCDA), all IEC 61850 Data Attribute Types (DATypes), IEC 61850 Data Types (DA's), Functional Constraints (FC), Presence Condition, Trigger Options and IEC 61850 Namespace. Based on a comparison between Figure 12 and Figure 13 we can observe the main difference between our proposed tool and conventional SCL validation tools respectively. For conventional tools, the database is manually generated from part 7 of the IEC 61850 standard making it too laborious and also prone to errors. Our proposed tool generates the data model database automatically and can be help automate the SCL validation process. This does not only minimize human-prone errors but also simplifies the use of the tool for substation automation or test engineers. 


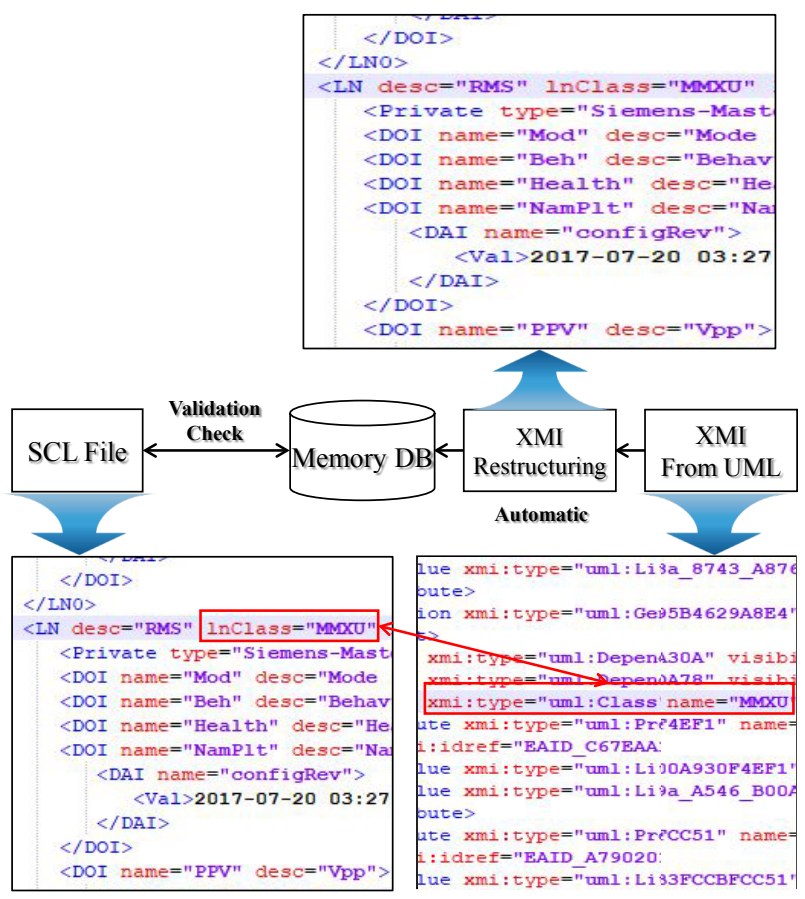

Figure 12. Proposed Algorithm for the IEC 61850 data model validation.

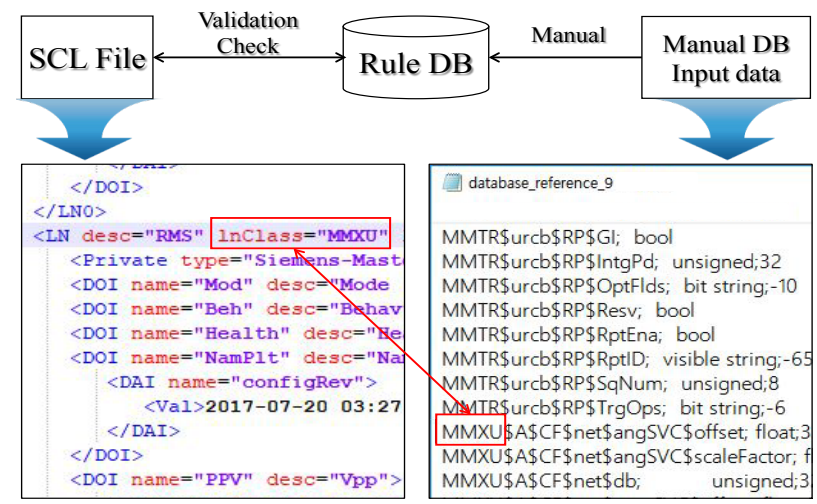

Figure 13. A practical illustration of the conventional SCL validation method.

\subsection{Generating the SCL Validation Rule}

Table 1 provides a list of the pre-defined validation rules utilized in the development of the proposed data model validator. These standardized rules are generated from the semantic requirements as specified in the UML data model. These rules are fixed and are ideally updated with each revision of the validation software. The main reason for adopting the rule-based approach is able to the test engineer utilizes the error message when debugging errors in the SCL file. The error message contains a summary which consists of an interactive hyperlink between the validation rule, the error type and the XML-based SCL line where the error occurred. This is in contrast to available validation tools which uses the "black-box method", essentially keeping all error related information 
Table 1. The list of rules created for SCL validation.

\begin{tabular}{|c|c|c|}
\hline Rule No. & Rule Categorization & Rule explanation \\
\hline Rule 1 & LN-Related validation Rule & $\begin{array}{l}\text { The lnClass in the LNodeType in the SCL must be consistent with the name of Logical Node } \\
\text { in the IEC } 61850 \text { UML Model }\end{array}$ \\
\hline Rule 2 & DO-Related Rule & The Mandatory DO defined in the SCL must be present in IEC 61850 UML Model \\
\hline Rule 3 & Presence Condition testing & $\begin{array}{l}\text { The Presence Condition of the Data Object (DO) in the SCL must be consistent with the } \\
\text { Presence Condition in the IEC } 61850 \text { UML Model for the specific DATA }\end{array}$ \\
\hline Rule 4 & DO name validation & $\begin{array}{l}\text { The Data Object (DO) name in the SCL must be consistent with the DO name of the IEC } \\
61850 \text { UML Model }\end{array}$ \\
\hline Rule 5 & $C D C$ name validation & $\begin{array}{l}\text { The Common Data Class (CDC) name of the DOType in the SCL must be consistent with } \\
\text { CDC naming convention in the IEC } 61850 \text { UML Model }\end{array}$ \\
\hline Rule 6 & & $\begin{array}{l}\text { The Data Attribute (DA) name and SDO name in the SCL DOtype must be consistent with } \\
\text { the standard naming convention in the IEC } 61850 \text { UML Model }\end{array}$ \\
\hline Rule 7 & $\begin{array}{l}\text { DA related Validation } \\
\quad \text { Rule }\end{array}$ & $\begin{array}{l}\text { The Mandatory Data Attribute (DA) and SDO in the SCL must be present in the IEC } 61850 \\
\text { UML Model }\end{array}$ \\
\hline Rule 8 & & $\begin{array}{l}\text { The DA type defined in the SCL must be consistent with the DA type in the IEC } 61850 \text { UML } \\
\text { Model for each specific DATA }\end{array}$ \\
\hline Rule 9 & Functional Constraint validation & $\begin{array}{l}\text { The Functional Constraint (FC) of all the Data Attributes (DA) in the SCL must be consistent } \\
\text { with the IEC } 61850 \text { UML Model }\end{array}$ \\
\hline Rule 10 & DA TrigOps Validation & $\begin{array}{l}\text { The Trigger operations (TrigOps) of the Data Attribute (DA) in the SCL must be consistent } \\
\text { with the IEC } 61850 \text { UML Model }\end{array}$ \\
\hline Rule 11 & EnumTypes Related Validation & $\begin{array}{l}\text { All EnumTypes defined in the SCL must be consistent with the EnumTypes defined in the } \\
\text { IEC } 61850 \text { UML Model }\end{array}$ \\
\hline Rule 12 & LNType-related Validation Rule & $\begin{array}{l}\text { The LN type used in SCL of the IED must be defined within the LNodeType of the } \\
\text { Data Type Templates in the UML Model }\end{array}$ \\
\hline Rule 13 & & $\begin{array}{l}\text { The DOI type used in the IED must be defined within the DOType of the UML Model } \\
\text { DataType Templates }\end{array}$ \\
\hline Rule 14 & DOType validation Rule & $\begin{array}{l}\text { The DAI type used in the IED must be defined in the DOType or DAType of the UML Model } \\
\text { DataTypeTemplates }\end{array}$ \\
\hline Rule 15 & EnumType related Validation Rule & $\begin{array}{l}\text { Any Enummerated Values (Enum) that is not defined in the EnumType of the } \\
\text { Data Type Templates of the UML Model must not be used }\end{array}$ \\
\hline Rule 20 & LNodeType related validation rule & $\begin{array}{l}\text { The name of DOI in the SCL under test must be consistent with the name of Data object in } \\
\text { LNodeType of the Standard UML model. }\end{array}$ \\
\hline Rule 21 & DOType related validation rule & $\begin{array}{l}\text { The name of DAI and SDI in the SCL under test must be consistent with the name of Data } \\
\text { Attribute in DOType in the standard UML model. }\end{array}$ \\
\hline Rule 22 & Value DAI Rule & $\begin{array}{l}\text { The value of DAI defined in the SCL under test must be within a range that is defined in the } \\
\text { standard IEC } 61850 \text { UML Model }\end{array}$ \\
\hline Rule 23 & EnumType Validation Rule & $\begin{array}{l}\text { The value of DAI in the SCL under test must be consistent with EnumType in the UML } \\
\text { model, if the type of DAI is EnumType }\end{array}$ \\
\hline Rule 24 & & Warning, If Duplicated definition of the same LNodeType is found in the SCL under test \\
\hline Rule 16 & Warning for LNodeType & $\begin{array}{l}\text { Warning, If the LNodeType defined in the DataTypeTemplates of the UML Model is not used } \\
\text { in the SCL under test }\end{array}$ \\
\hline Rule 25 & & Warning, If Duplicated definition of the same DOType is found in the SCL under test \\
\hline Rule 17 & Warning for DOType & $\begin{array}{l}\text { Warning, If the DOType defined in the DataTypeTemplates of the UML Model is not used in } \\
\text { the SCL under test }\end{array}$ \\
\hline Rule 26 & & Warning, If Duplicated definition of the same DAType is found in the SCL under test \\
\hline Rule 18 & Warning for DAType & $\begin{array}{l}\text { Warning, If the DAType defined in the DataTypeTemplates of the UML Model is not used in } \\
\text { the SCL under test }\end{array}$ \\
\hline Rule 27 & & Warning, If Duplicated definition of the same EnumType is found in the SCL under test \\
\hline Rule 19 & Warning for Enum Type & $\begin{array}{l}\text { Warning, If the EnumType defined in the DataTypeTemplates of the UML Model is not used } \\
\text { in the SCL under test }\end{array}$ \\
\hline
\end{tabular}


hidden except for the XML-based SCL line where the error occurred. This might not be enough for an informed error correction and this is one of the problems our proposed tool can mitigate. Also although, rules are fixed and are ideally updated with each revision of the validation software, we are working to incorporate an user-defined rule generation functionality in the future in order to ensure flexibility.

\section{Practical Implementation of IEC 61850 Data Model}

\subsection{Main Features of UML-Based SCL Validation S/W}

Figure 14(a) shows the pre-defined UML based validation test rule. The rules are generated based on the semantic specifications of IEC 61850. By selecting a specific rule from the list of validation rules, a specific aspect of the SCL data model is checked for errors using the UML memory database. The display technique used in Figure 14(b) helps the test engineer to understand the various standard data objects. This is implemented using the standard UML model of IEC 61850-7 as explained earlier. It shows the detailed information about the data objects in the SCL file thereby making it easy for test engineers to understand the relationship between different logical nodes and data objects without the need to constantly refer to the standard as it is quite voluminous.

\subsection{Test Case for UML-Based SCL File Validation}

\subsubsection{SCL Validation Test scenario}

The scenario under consideration is to test for the implementation of the Logical node type element according to the IEC 61850-7 standard. The LNodeTypes are provided under the DataType template section of the SCL file. It describes the instantiation of a specific logical node. Thus, it contains information including the data objects (DO), the various data attributes (DA) and the configuration default parameters. The data object in an LNodeType becomes a Data Object Instance (DOI) in the corresponding logical node. As stated in Table 2 and in accordance with part 7 of the IEC 61850 standard the following are the attributes of the LNodeType element.

These attributes can be observed in the schema extract defined in part 6 of the IEC 61850, illustrated in Figure 15.

To implement a tractable test case for the data model validation, the SCL file is edited as can be seen in Figure 16.

The wrong Inclass (XCBR is witched for XSWI) and DOType descriptions are provided for the XCBR logical node case. Figure 17 presents the validation rules implemented to test the above-mentioned error scenario in the SCL file.

\subsubsection{Test Results and SCL File Editing}

The test results for the data model validation are displayed with the corresponding error guide as seen in Figure 18. The error guide shows the XML line number where the error occurred. This makes debugging easy for the test engineer. A rule highlighted in red shows an error while that highlighted in yellow indicate a 
Table 2. Example of attributes of LNodeType element for testing.

\begin{tabular}{cc}
\hline Element & Explanation \\
\hline id & The identification tag for the LNodeType in the SCL file \\
InClass & The logical node base class as defined in the IEC 61850-7-3 \\
DOname & The data object name as defined in IEC 61850-7-4 \\
DOType & The data object type which references the id of the DOType definition. \\
\hline
\end{tabular}

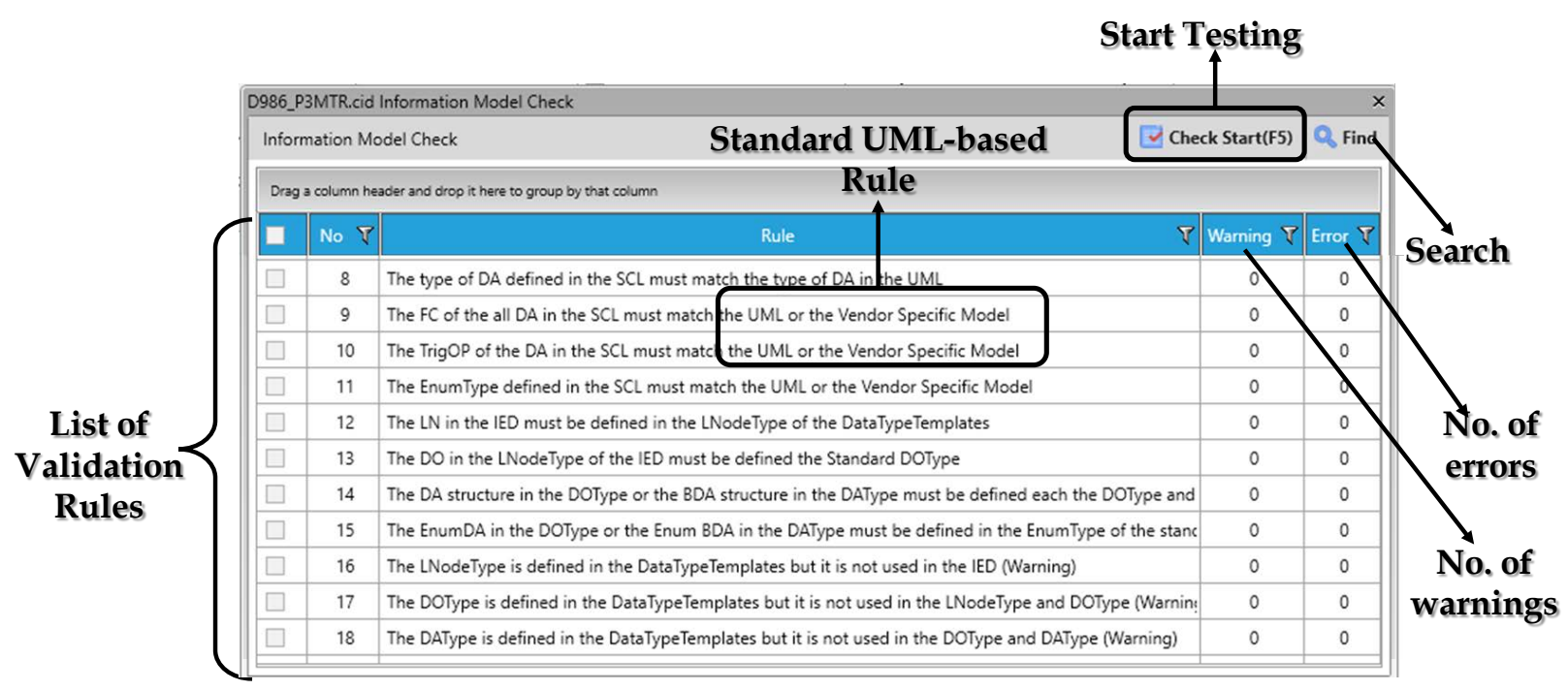

(a)

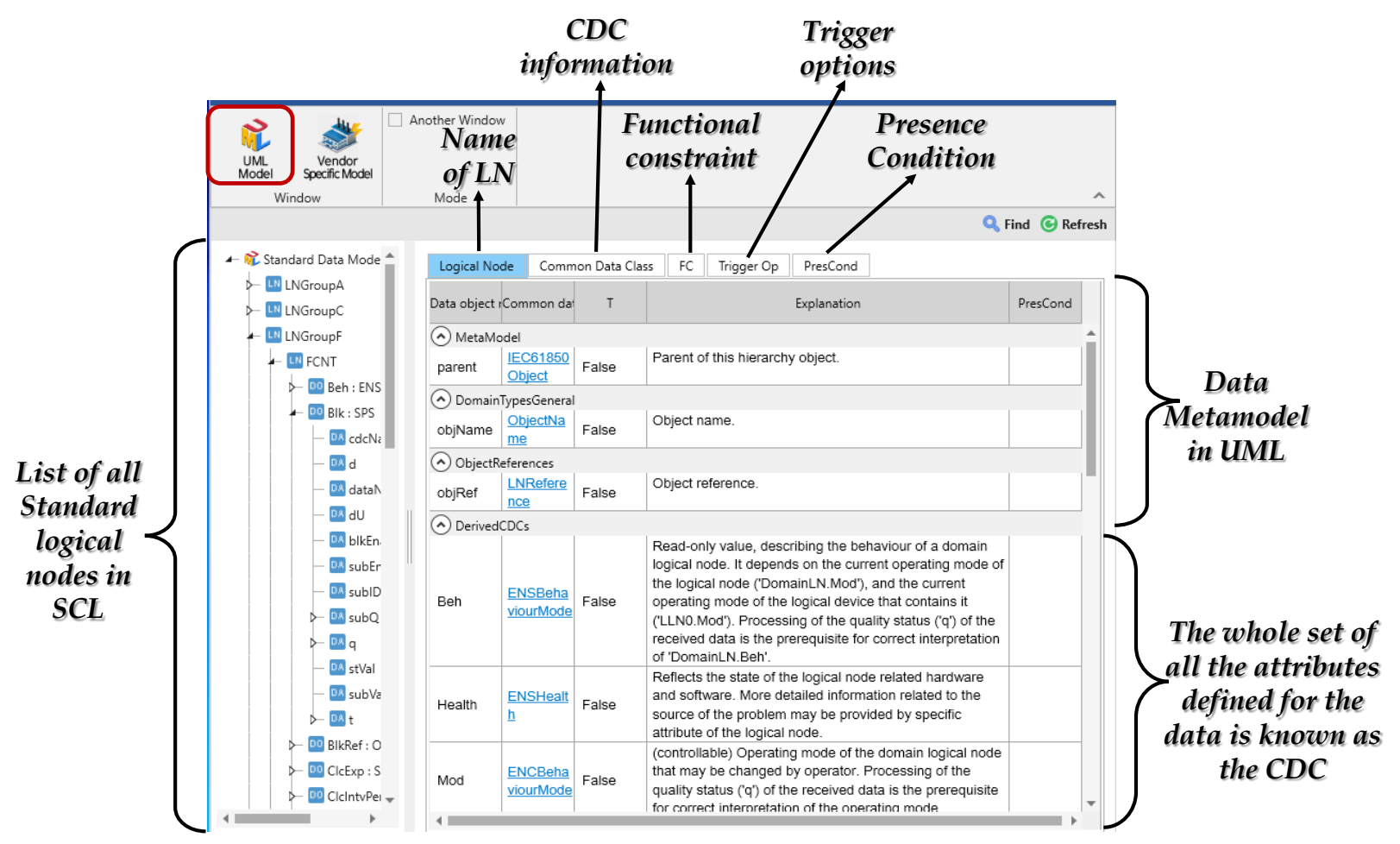

(b)

Figure 14. (a) Section of the proposed Data model validation rules; (b) Standard data model extracted from the SCL. 


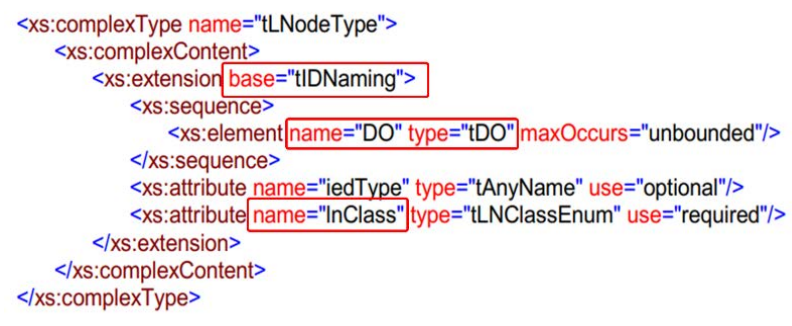

Figure 15. Schema extract for the LNodeType element [25].

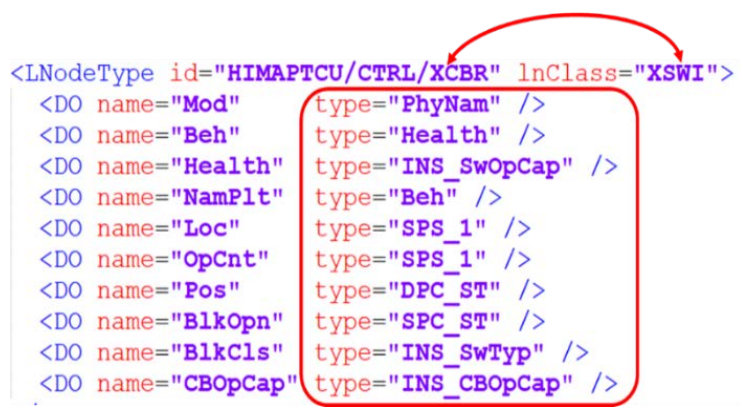

Figure 16. Extract of SCL (under test) XCBR LNodeType data for testing.

\begin{tabular}{|c|c|c|c|c|c|}
\hline \multirow{13}{*}{$\begin{array}{c}\text { Standard } \\
\text { data model } \\
\text { validation } \\
\text { test rule }\end{array}$} & \multicolumn{5}{|c|}{ D986_P3MTR.cid Information Model Check } \\
\hline & \multicolumn{3}{|c|}{ Information Model Check } & $\nabla$ Check Start(F5) & Q Find \\
\hline & \multicolumn{5}{|c|}{ Drag a column header and drop it here to group by that column } \\
\hline & a & No $\checkmark$ & Rule & Warning $?$ & Error $\nabla$ \\
\hline & $\square$ & 1 & The InClass in the LNodeType must exist (be present) in the UML or the Vendor Specific Model & 0 & 0 \\
\hline & $\nabla$ & 2 & The Mandatory DO defined in the UML or the Vendor Specific Model must exist (be present) in the SCL & 0 & 0 \\
\hline & $\nabla$ & 3 & All DO and DA must be associated with the correct "Presence Condition" & 0 & 0 \\
\hline & $\nabla$ & 4 & The DO under LNodeType in the SCL must be consistent with the UML or the Vendor Specific Model & 0 & 0 \\
\hline & $\square$ & 5 & The CDC of the DOType in the SCL must match the definition in the UML or in the Vendor Specific Model & 0 & 0 \\
\hline & $\square$ & 14 & The DA structure in the DOType or the BDA structure in the DAType must be defined each the DOType and $c$ & 0 & 0 \\
\hline & $\square$ & 15 & The EnumDA in the DOType or the Enum BDA in the DAType must be defined in the EnumType of the standi & 0 & 0 \\
\hline & $\nabla$ & 16 & The LNodeType is defined in the DataTypeTemplates but it is not used in the IED (Warning) & 0 & 0 \\
\hline & $\square$ & 17 & The DOType is defined in the DataTypeTemplates but it is not used in the LNodeType and DOType (Warning:) & 0 & 0 \\
\hline
\end{tabular}

Figure 17. Selected Validation rules for SCL (XCBR LNodeType) data testing.

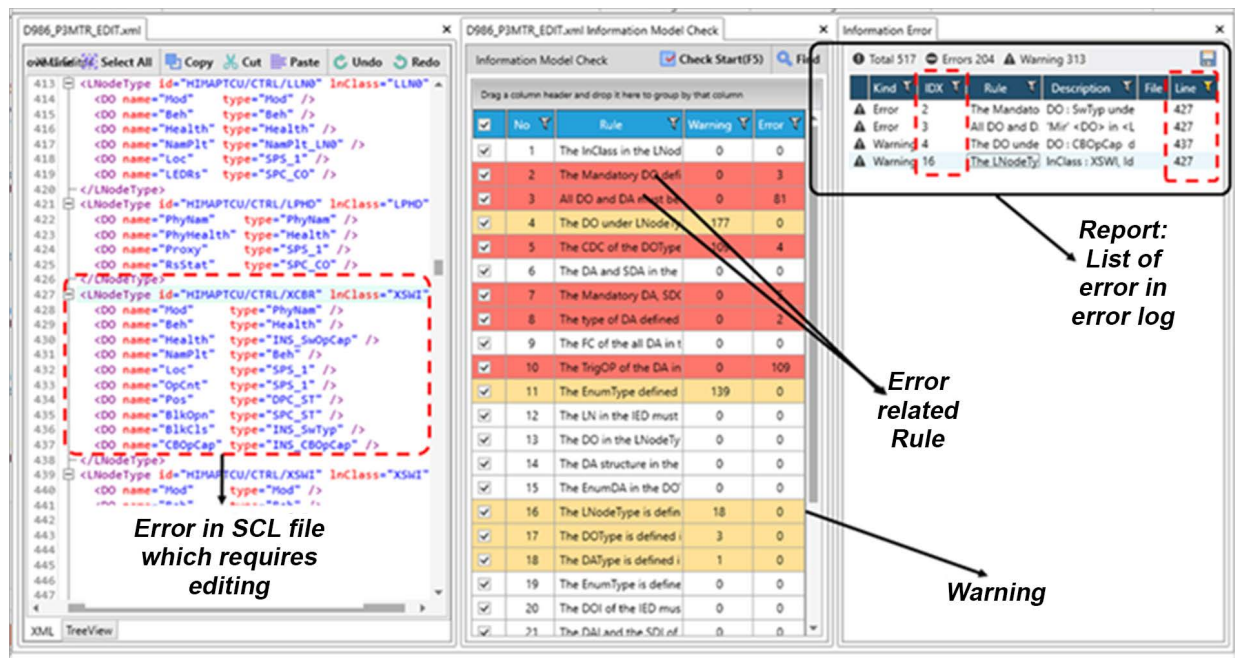

Figure 18. Results for the data model validation. 
warning. It should be noted that warnings usually do not require debugging as much as the errors do. It can be observed that the error occurred in line 427 of the SCL file, where the initially edit was made. The wrong LNodeType configuration goes against the UML model rule which stipulates that "all mandatory DO defined in the UML model must be present in the SCL". The final stage of the validation process is checking editing the SCL file in case of errors.

By selecting the error in the error log, the test engineer can edit the SCL file to achieve consistency with the standard. After editing the SCL file as shown in Figure 19, it is recommended to run a new test to validate the changes and to establish that the SCL file under test has no errors which can affect communication in the practical substation scenario.

\subsection{Merits of the Advanced SCL Validation Tool}

There are several benefits of the above proposed SCL validation tool. In the first instance, the proposed data model validation of the SCL file can check the standard data model using the UML model. The standard data model is quite large and described using tables in several parts of the IEC 61850 standard. This makes tracking, understanding and validating the SCL file for data model related errors quite difficult for the test engineer. With the use of the UML model is quite comprehensive making it easier for the test engineer to implement accurate validation of the SCL file. The proposed validation tool for IEC 61850 SCL files employs the rule-based validation data model validation. For most conventional validation tools, the validation rules are not shown in the user interface and therefore it is difficult to understand the main reason behind an error. The rule-based validation makes it easy to understand the various aspects of the SCL required for validation. In case of an error, the test engineer can easily identify the cause of the error. It is also easy to directly debug the SCL file using the error guide without the need for third party XML editors. Our proposed algorithm is easy to use because it implements an automated checking process without the need of consistent check up by the test engineer which can cause lots of delay. The test process is top-bottom validation with the results listing out all the test errors while in the conventional validation case, each error causes the test to stop so that the test engineer can correct the error.

\section{Conclusions}

In order to integrate multiple heterogeneous devices in a single communication network in a smart grid-based power grid system, the use of the standard data model in the system becomes very important. It is not easy for existing system engineers to correctly use the vast data model required in the new digital environment. Also the IEC 61850 standard data model continues to grow as the number of data standard models continues to expand from the substation to other domain, such as including IEC 61400-25 (wind power generation) and IEC 61850-7-410 (hydroelectric power generation). 


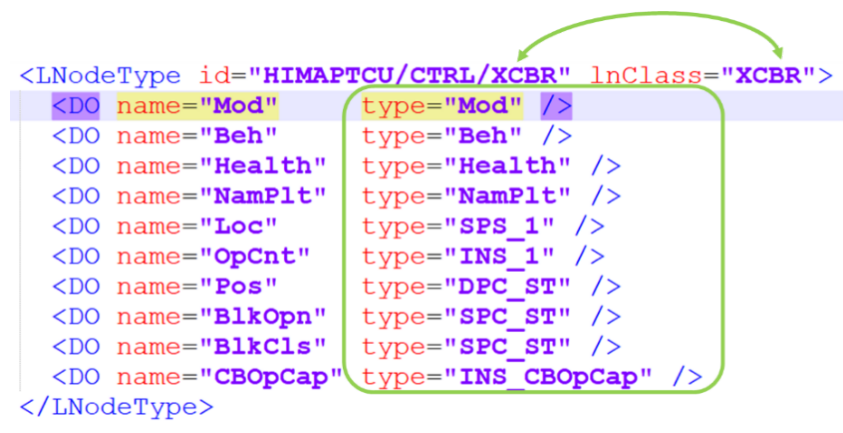

Figure 19. Illustration of the debugging process used to edit the SCL file.

In this paper, we propose a new test technique that can detect and correct the use and conformance of the standard data model of the multiple devices from different vendors during the digital substation engineering process when the various IEC 61850-based IEDs are being installed in the field of the power grid system. We have also demonstrated the detailed implementation of the proposed algorithm using an SCL validation developed by KEPCO. The proposed SCL validation tool can present the substation engineer with the ability to separately validate both the standard data model.

Our proposed tool is unique due to the fact that it is equipped with both the error guide and the rule guide which serves as a means to fully understand and debug errors associated with non-standard data model with the use of the SCL editor. The error guide also known as the SCL editor guide helps the test engineer to successfully align an SCL validation error to the validation rule and the corresponding XML line where the error occurred. This will help to solve interoperability problems which occur in practical SA systems by ensuring the syntactic and semantic correctness of the multi-vendor SCL files.

Future research would focus on improving the proposed validation tool by implementing other validation techniques including flexible user defined rule, vendor specific data extension validation and schema based validation.

Finally, although the use of the relational database model is quite flexible and it is capable of removing all redundancies and thereby ambiguities from the data stored, it has some strong drawbacks like its inability to support schema evolution and also some weakness as a result of mismatches between the object oriented and relational database world. We therefore hope to use non-relational database in the future in order to benefit fully from the UML Model.

In the future, it is expected that the SCL file would serve as the sole configuration file for all the various domain of the electrical power system including distribution automation, renewable energy, vehicle-to-grid etc. Therefore, to ensure interoperability in modern digital substation automation systems, our proposed SCL validator is required.

\section{Conflicts of Interest}

The authors declare no conflicts of interest regarding the publication of this paper. 


\section{References}

[1] Pagani, G.A. and Aiello, M. (2013) The Power Grid as a Complex Network: A Survey. Physica A: Statistical Mechanics and Its Applications, 392, 2688-2700. https://doi.org/10.1016/j.physa.2013.01.023

[2] Roostaee, S., Hooshmand, R. and Ataei, M. (2011) Substation Automation System Using IEC 61850. Proceedings of the 5 th International Power Engineering and Optimization Conference, Shah Alam, Selangor, 6-7 June 2011, 393-397.

[3] Brand, K.P. and Janssen, M. (2005) The Specification of IEC 61850 Based Substation Automation Systems. Paper Presented at the DistribuTech 2005, San Diego, 25-27 January 2005, 8 p. https://library.e.abb.com/public/b0dc46b17050d26bc125705a004de6d5/Brand_Janss en_DistribuTech2005.pdf

[4] Adamiak, M., Baigent, D. and Mackiewicz, R. IEC 61850 Communication Networks and Systems in Substations: An Overview for Users.

http://www.gegridsolutions.com/multilin/journals/issues/spring09/iec61850.pdf

[5] Mackiewicz, R.E. (2006) Overview of IEC 61850 and Benefits. Proceedings of the IEEE/PES Transmission and Distribution Conference and Exhibition, Dallas, TX, 21-24 May 2006, 376-383. https://doi.org/10.1109/TDC.2006.1668522

[6] Wagh, K.S. and More, A. (2015) Comparative Analysis of IEC 61850 Edition-I and II Standards for Substation Automation. Proceedings of the 2015 IEEE International Conference on Computational Intelligence and Computing Research (ICCIC), Madurai, 10-12 December 2015, 1-6. https://doi.org/10.1109/ICCIC.2015.7435756

[7] Ozansoy, R., Zayegh, A. and Kalam, A. (2009) Object Modeling of Data and DataSets in the International Standard IEC 61850. IEEE Transactions on Power Delivery, 24, 1140-1147. https://doi.org/10.1109/TPWRD.2008.2005658

[8] Marcadet and Lambert, E. (2016) RiseClipse: Why Working at the Model Level Is Better for Validating Data Conforming to IEC Standards. Proceedings of the 2016 Power Systems Computation Conference (PSCC), Genoa, 20-24 June 2016, 1-7.

[9] Peng, X., Liang, Y.C., Luo, Z.P., Pan, F.L. and Li, L. (2016) Analyses and Comparisons of SCL Files in Substation Configurator. Proceedings of the 3 rd International Conference on Systems and Informatics (ICSAI), Shanghai, 19-21 November 2016, 297-300.

[10] Huo, Z.H., Zhang, L.M. and Zhang, Z.X. (2008) Research on Graphics Model Design for IEC61850 SCL Visual Configuration. Proceedings of IEEE Pacific-Asia Workshop on Computational Intelligence and Industrial Application, Wuhan, 19-20 December 2008, 700-704. https://doi.org/10.1109/PACIIA.2008.42

[11] Pan, Y., Sun, K.H. and Ma, L.J. (2012) Design and Implementation of Visual SCL Configurator. Proceedings of the International Conference on Automatic Control and Artificial Intelligence, Xiamen, 3-5 March 2012, 782-785.

[12] Beil, L. and Lian-shunl, M. (2006) To Realize the SCL Configurator of IEC6 1850 Based on Relative Model. Proceedings of the International Conference on Power System Technology, Chongqing, 22-26 October 2006, 1-7. https://doi.org/10.1109/ICPST.2006.321870

[13] Yang, Y., et al. (2014) Comparison Method for Differentiation of IEC 61850 Based Substation Configuration Files. Proceedings of the International Conference on Power System Technology, Chengdu, 20-22 October 2014, 1796-1801. https://doi.org/10.1109/POWERCON.2014.6993635

[14] Konig, J., Zhu, K., Nordstrom, L., Ekstedt, M. and Lagerstrom, R. (2010) Mapping 
the Substation Configuration Language of IEC 61850 to ArchiMate. Proceedings of the 14th IEEE International Enterprise Distributed Object Computing Conference Workshops, Vitoria, 25-29 October 2010, 60-68. https://doi.org/10.1109/EDOCW.2010.35

[15] Hou, S.Z., Liu, W. and Kong, F.Y. (2013) IEC61850 Gateway SCL Configuration Document Research. Proceedings of the 3 rd International Conference on Intelligent System Design and Engineering Applications, Hong Kong, 16-18 January 2013, 824-831. https://doi.org/10.1109/ISDEA.2012.196

[16] Vetter, C., Frei, C. and Obrist, M. (2008) Validating a Standardized Configuration Description. U.S Patent 8156061B2.

[17] DNV GL, UniCA SCL (2017) Checker Version 3.29.

[18] Jang, B.-T., Alidu A. and Kim, N. (2017) Design of an Algorithm for the Validation of SCL in Digital Substations. KEPCO Journal on Electric Power and Energy, 3, 89-97.

[19] Kostic, T., Preiss, O. and Frei, C. (2005) Understanding and Using the IEC 61850: A Case for Meta-Modelling. Computer Standards \& Interfaces, 27, 679-695.

[20] Zhu, Y.L., Wang, D.W., Wang, Y. and Zhao, W.Q. (2009) Study on Interoperable Exchange of IEC 61850 Data Model. Proceedings of the 4th IEEE Conference on Industrial Electronics and Applications, Xi'an, 25-27 May 2009, 2724-2728. https://doi.org/10.1109/ICIEA.2009.5138698

[21] (2013) Communication Networks and Systems for Power Utility Automation Part 7-3: Basic Communication Structure-Common data classes, IEC 61850 Standard.

[22] (2013) Communication Networks and Systems for Power Utility Automation Part 7-4: Basic communication structure-Compatible Logical Node Classes and Data Object Classes, IEC 61850 Standard.

[23] Apostolov, A.P. (2010) UML and XML Use in IEC 61850. IEEE PES T\&D 2010, New Orleans, LA, USA, 19-22 April 2010, 1-6. https://doi.org/10.1109/TDC.2010.5484325

[24] Kim, N.D., Alidu, A., Jang, B.T., Lee, N.H. and Yun, Y.B. (2017) Using UML Data Model for SCL Validation. Proceedings of the KIEE-The Korean Society of Power Engineering Proceedings Spring Conference on Power Systems, Hyatt Hotel Jeju South Korea, 23-25 March 2017, 229-231.

[25] (2013) Communication Networks and Systems for Power Utility Automation Part 6: Configuration Description Language for Communication in Electrical Substations Related to IEDs, IEC 61850 Standard Part 6.

[26] Young, P. and Stevens, J. Consistency and Completeness Checking of 61850 SCL Files for Compliance and Interoperability.

http://trianglemicroworks.com/docs/default-source/referenced-documents/consiste ncy-and-completeness-checking-of-61850-scl-files.pdf?sfvrsn $=4$

[27] Stephen Gerspach, C.V. and Maeda, T. (2006) Intelligent Electronic Device Configuration Inspection. European Patent No. US8165841B2, ABB Zurich. https://patentimages.storage.googleapis.com/24/28/a9/76c9a98ce230fe/US8165841. pdf 


\section{Abbreviations}

\begin{tabular}{ll} 
ACSI & Abstract Communication Service Interface \\
CDC & Common Data Classes \\
CID & Configured IED Description \\
GOOSE & Configured IED Description \\
HMI & Human Machine Interface \\
ICD & Configured IED Description \\
IEC & International Electro-technical Commission \\
IED & Intelligent Electronic Device \\
IP & Internet Protocol \\
ISO & International Standards Organization \\
LAN & Local Area Network \\
LD & Logical Device \\
LN & Logical Node \\
MMS & Manufacturing Message Specification \\
OSI & Open System Interconnection \\
SA & Substation Automation \\
SAS & Substation Automation Systems \\
SCADA & Supervisory Control and Data Acquisition \\
SCD & Substation Configuration Description \\
SCL & Substation Configuration description Language \\
SCSM & Specific Communication Service Mappings \\
SMV & Samples Measured Values \\
SSD & System Specification Description \\
TCP/IP & Transmission Control Protocol over Internet Protocol \\
UCA & Utility Communication Architecture \\
XML & Extensible Markup Language \\
\hline
\end{tabular}

